\title{
Positron Emission Tomography in Subcutaneous Panniculitis-Like T-Cell Lymphoma
}

\author{
Victor R. Rodriguez, MD, MS, ${ }^{1}$ Aparna Joshi, MD, ${ }^{1}$ Fangyu Peng, MD, ${ }^{1}$ Raja M. Rabah, $\mathrm{MD}^{2}$ \\ Paul T. Stockmann, $\mathrm{MD}^{3}$ and Süreyya Savaşan, $\mathrm{MD}^{4}{ }^{4}$
}

\begin{abstract}
Subcutaneous panniculitis-like T-cell lymphoma (SPTCL), an uncommon disorder, was diagnosed in a 17-year-old female when she presented with multiple hard subcutaneous masses that developed over 3 years. She was treated on chemotherapy consisting of cyclophosphamide, doxorubicin, vincristine, and prednisone. Pre-
\end{abstract}

and post-treatment positron emission tomography study demonstrated dramatic resolution of the subcutaneous lesions indicating its usefulness in SPTCL staging and treatment response monitoring. Pediatr Blood Cancer 2009;52:406-408. 두 2008 Wiley-Liss, Inc.

Key words: CHOP chemotherapy; positron emission tomography; subcutaneous panniculitis-like T-cell lymphoma

\section{INTRODUCTION}

Subcutaneous panniculitis-like T-cell lymphoma (SPTCL) is a rare type of cutaneous lymphoma and is classified as a post-thymic lymphoproliferative disorder that belongs to a new subset of mature, peripheral T-cell neoplasms by the World Health Organization (WHO) [1]. The disease is characterized clinically by an indolent course of recurrent, self-healing, or progressive subcutaneous nodules. These nodules both clinically and histologically resemble panniculitis. Alternatively, a rapidly progressive course can be seen with subcutaneous nodules accompanied by constitutional symptoms and, in some cases, the development of a potentially fatal hemophagocytic syndrome with significant cytopenia [2]. Here we report a teenager with SPTCL whose disease was studied by positron emission tomography (PET) at the time of diagnosis and after completion of the treatment.

\section{CASE HISTORY}

A 17-year-old African American female presented with multiple subcutaneous hard masses throughout her body that started in her left lower back approximately 3 years ago. Since then, the original lesion has grown in size and new lesions appeared throughout her body, which have also enlarged in time. She did not have any history of fever, weight loss, night sweats, or pruritus. On examination, the subcutaneous lesions were flat, minimally depressed, and hard with irregular borders located on the left lower back, right anterior abdominal wall, bilateral thighs, right arm, and right cheek measuring up to $4 \mathrm{~cm} \times 4 \mathrm{~cm}$ in size. The lesions were not tender, and there was no change in the overlying skin or hypoesthesia. The remainder of her physical exam was otherwise unremarkable.

She had iron deficiency anemia with hemoglobin of $10.5 \mathrm{~g} / \mathrm{dl}$, increased serum IgG level at $3,120 \mathrm{mg} / \mathrm{dl}$, elevated erythrocyte sedimentation rate (ESR) at $112 \mathrm{~mm} / \mathrm{hr}$, low T lymphocyte count at 650/mcl with normal CD4/CD8 ratio, and high titers against EBV; VCA-IgM was negative, VCA-IgG 1:640, EA-IgG >1:5,120, EBNA >1:320. Her ANA, HIV serology, and EBV PCR were negative and LDH was within normal limits.

Biopsy of abdominal wall mass showed subcutaneous fat necrosis and diffuse infiltration by pleomorphic lymphoid cells mixed with histiocytes. Many mitotic figures and karyorrhexis were present. Atypical lymphocytes with hyperchromatic nuclei were noted surrounding necrotic adipocytes. Immunohistochemistry revealed that the atypical lymphocytes stained positively with
T cell markers including CD3, CD4, CD5, and CD8. They were negative for CD2, CD30, Alk1, CD56, CD20, EBV latent membrane protein-1 (LMP-1), and Tia1 staining and EBV in situ hybridization. Karyotype analysis of the tumor tissue was not successful due to lack of mitotic figures. T cell receptor (TCR)-gamma was negative by PCR. However, TCR-beta rearrangement was seen in Eco R1 enzyme digest, but this was not sufficient to account for clonality. Bone marrow biopsy was negative for lymphoma involvement and karyotype showed 46, XX. Cerebrospinal fluid did not reveal the presence of lymphoma cells.

A contrast-enhanced computed tomography (CT) of the neck and chest demonstrated a $3.6 \mathrm{~cm} \times 1.6 \mathrm{~cm}$ soft tissue lesion in the subcutaneous tissue of the right face. Several prominent lymph nodes measuring up to $1.2 \mathrm{~cm}$ in short axis diameter were present in both axilla. Magnetic resonance imaging (MRI) evaluation of the abdomen and pelvis with gadolinium contrast demonstrated multiple lesions in the subcutaneous tissues of the abdomen and pelvis that were heterogeneous in signal on T1-weighted images and increased signal peripherally on T2-weighted images. The subcutaneous lesions enhanced peripherally after the administration of gadolinium contrast. PET using 2-deoxy-2-[F-18]-fluoro-D-glucose (FDG), which was performed as described previously [3], demonstrated numerous foci of abnormal FDG uptake involving the subcutaneous tissues throughout the body and axillary lymph nodes without any visceral involvement. The maximum standardized uptake values (SUV) of FDG uptake by these lesions varied from 2.6 to 4.3 .

\footnotetext{
${ }^{1}$ Division of Radiology, Carman and Ann Adams Department of Pediatrics, Children's Hospital of Michigan, Wayne State University School of Medicine, Detroit, Michigan; ${ }^{2}$ Division of Pathology, Carman and Ann Adams Department of Pediatrics, Children's Hospital of Michigan, Wayne State University School of Medicine, Detroit, Michigan; ${ }^{3}$ Division of Surgery, Carman and Ann Adams Department of Pediatrics, Children's Hospital of Michigan, Wayne State University School of Medicine, Detroit, Michigan; ${ }^{4}$ Division of Hematology/Oncology, Carman and Ann Adams Department of Pediatrics, Children's Hospital of Michigan, Wayne State University School of Medicine, Detroit, Michigan

*Correspondence to: Süreyya Savaşan, 3901 Beaubien Blvd., Detroit, MI 48201. E-mail: ssavasan@med.wayne.edu
}

Received 18 May 2008; Accepted 9 September 2008
(C) 2008 Wiley-Liss, Inc.

DOI 10.1002/pbc.21805

Published online 4 November 2008 in Wiley InterScience (www.interscience.wiley.com) 

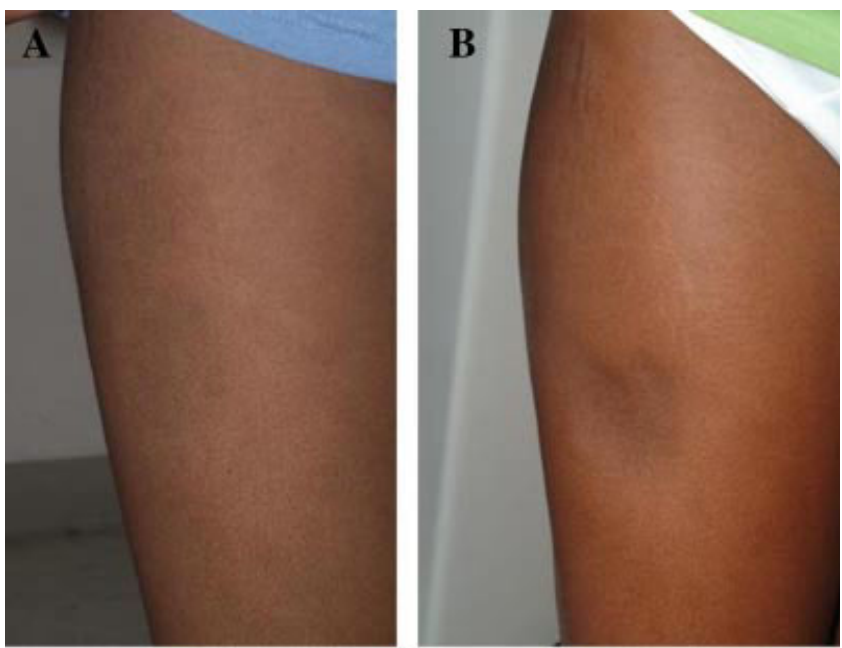

Fig. 1. Right anterior leg lesion shows further depression following chemotherapy. A: At the time of diagnosis and (B) after the completion of chemotherapy. [Color figure can be viewed in the online issue, which is available at www.interscience.wiley.com.]

The patient was treated on CHOP chemotherapy consisting of cyclophosphamide $\left(750 \mathrm{mg} / \mathrm{m}^{2} /\right.$ dose $)$, doxorubicin $\left(50 \mathrm{mg} / \mathrm{m}^{2} /\right.$ dose), vincristine $\left(1.4 \mathrm{mg} / \mathrm{m}^{2} /\right.$ dose $)$, and prednisone (100 mg/day for 5 days) for a total of six courses. There was a remarkable response with resolution of the nodularity in the lesions that, however, was associated with hardening of the subcutaneous tissues and further depression at lesion sites (Fig. 1). The repeat PET study revealed complete resolution of abnormal FDG uptake in the involved sites (Fig. 2).

One year after the completion of chemotherapy, the patient continues to be doing well without any evidence of recurrence. Despite significant improvement, she continues to have increased serum IgG level at $1,990 \mathrm{mg} / \mathrm{dl}$, elevated ESR at $32 \mathrm{~mm} / \mathrm{hr}$, low
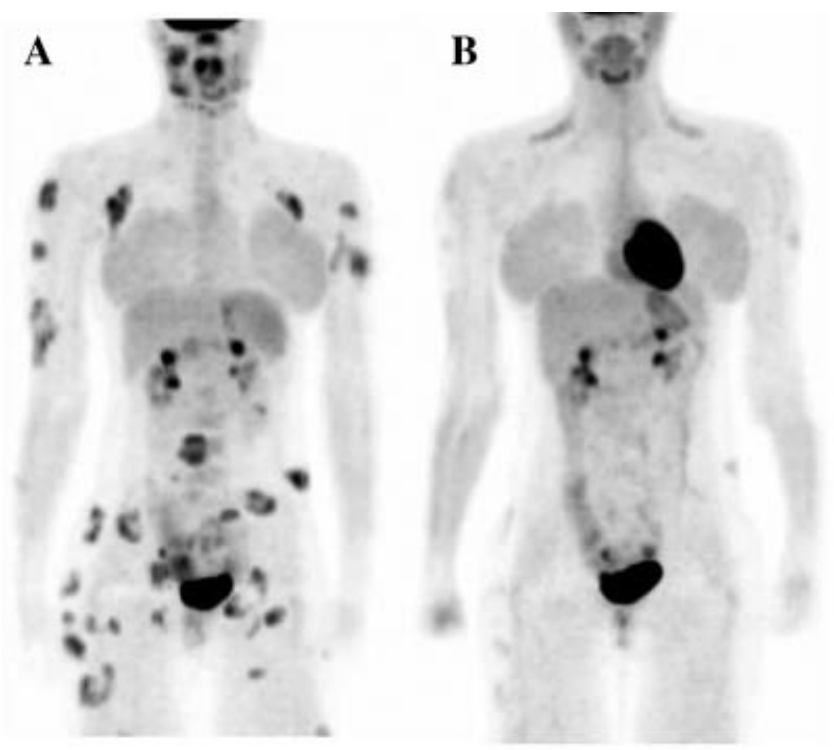

Fig. 2. Complete resolution of abnormal FDG uptake by the subcutaneous lesions after chemotherapy. A: At the time of diagnosis and (B) after the completion of chemotherapy.
T lymphocyte count at $816 / \mathrm{mcl}$ with normal CD4/CD8 ratio and high EBV titers with negative VCA-IgM and positive VCA-IgG at 1:320, EA 1:2,560, and EBNA >1:320.

\section{DISCUSSION}

SPTL is characterized by infiltration of the subcutaneous tissue by pleomorphic $\mathrm{T}$ cells and benign macrophages that mimic lobular panniculitis. The pertinent histologic features described by the WHO include neoplastic T cells with preferential involvement of the subcutaneous tissue with relative sparing of overlying epidermis and dermis, rimming of neoplastic cells surrounding individual fat cells, and clonal TCR gene rearrangement. This disorder is identified in almost equal numbers of male and females, rarely in children and the median age of diagnosis is 39 years [4-6]. Cases with $\alpha / \beta$ T cell subtype have much superior outcome compared with $\gamma / \delta$ T cell subtype, and it was proposed that these subcategories may represent different pathologies [7-8].

Presence of hemophagocytosis in some cases [2,8], development of cases after solid organ transplantation [9], occasional spontaneous resolution of lesions, and full recovery of certain cases following immunosuppressive therapy, particularly with $\alpha / \beta \mathrm{T}$ cell subtype strongly suggest a component of immune dysregulation in this disorder. Our patient, most likely to have $\alpha / \beta$ T cell subtype had several laboratory evidence of associated immune aberration with elevated serum IgG level, T lymphocytopenia, and very high EBV titers without EBV-PCR and EBV-related LMP-1 expression in tumor cells. She responded to $\mathrm{CHOP}$ chemotherapy very well with improvement in her serum IgG, ESR, T lymphocyte count, and decrease in EBV EA titer, but without complete normalization, 1 year after the completion of chemotherapy. This may indicate underlying immune aberration in SPTCL.

The use of ultrasound and Gallium-67 scintigraphy has been reported in SPTCL [10-11]. The CT of the neck and chest and MRI of the abdomen and pelvis showed the presence of subcutaneous lesions and small lymph nodes in our case. However, PET study was very sensitive to be able to show all the lesions including the ones palpated on physical examination and resolution of abnormal FDG uptake in the involved sites following chemotherapy. In primary cutaneous non-Hodgkin lymphoma, PET was shown to be useful for initial staging and restaging following therapy [12]. Three of five primary cutaneous anaplastic large cell lymphoma cases were negative by PET, whereas a single patient with SPTCL was positive in a different retrospective study [13]. Although there is need for further study, the findings in our case also suggest that PET provides valuable information in detecting lesions in SPTCL and may be useful in disease staging and monitoring treatment response.

\section{REFERENCES}

1. Jaffe ES, Ralfkiaer E. Subcutaneous panniculitis like T-cell lymphoma. In: Jaffe ES, Harris NL, Stein H, Vardiman JW, editors. World Health Organization classification of tumors: Pathology and genetics of tumors of hematopoietic and lymphoid tissues. Lyon, France: IARC Press; 2001. pp. 212-215.

2. Papenfuss JS, Aoun P, Bierman PJ, et al. Subcutaneous panniculitis-like T-cell lymphoma: Presentation of 2 cases and observations. Clin Lymphoma 2002;3:181-183.

3. Peng F, Rabkin G, Muzik O. Use of 2-deoxy-2-[F-18]-fluoro-Dglucose positron emission tomography to monitor therapeutic 
response by rhabdomyosarcoma in children: Report of a retrospective case study. Clin Nucl Med 2006;31:394-397.

4. Go RS, Wester SM. Immunophenotypic and molecular features, clinical outcomes, treatments and prognostic factors associated with subcutaneous panniculitis-like T-cell lymphoma. Cancer 2004;101:1404-1413.

5. Yim JH, Kim M-Y, Kim HO, et al. Subcutaneous panniculitis-like T-cell lymphoma in a 26-month-old child with a review of the literature. Pediatr Dermatol 2006;23:537-540.

6. Windsor R, Stiller C, Webb D. Peripheral T-cell lymphoma in childhood: Population-based experience in the United Kingdom over 20 years. Pediatr Blood Cancer 2008;50:784-787.

7. Hoque SR, Child FJ, Whittaker SJ, et al. Subcutaneous panniculitis-like T-cell lymphoma: A clinicopathological, immunophenotypic and molecular analysis of six patients. Br J Dermatol 2003; 148:516-525.

8. Willemze R, Jansen PM, Cerroni L, et al. Subcutaneous panniculitis-like T-cell lymphoma: Definition, classification, and prognostic factors: An EORTC Cutaneous Lymphoma Group Study of 83 cases. Blood 2008;111:838-845.

9. Bregman SG, Yeaney GA, Greig BW, et al. Subcutaneous panniculitic T-cell lymphoma in a cardiac allograft recipient. J Cutan Pathol 2005;32:366-370.

10. Hung GD, Chen YH, Chen DY, et al. Subcutaneous panniculitislike T-cell lymphoma presenting with hemophagocytic lymphohistiocytosis and skin lesions with characteristic high-resolution ultrasonographic findings. Clin Rheumatol 2007;26:775778.

11. Shen L, Alam-Fotias S, Mansberg R, et al. Subcutaneous panniculitis-like $\mathrm{T}$ cell lymphoma demonstrated on Gallium-67 scintigraphy. Clin Nucl Med 2005;30:500-502.

12. Kumar R, Xiu Y, Zhuang HM, et al. 18F-fluorodeoxyglucosepositron emission tomography in evaluation of primary cutaneous lymphoma. Br J Dermatol 2006;155:357-363.

13. Kako S, Izutsu K, Ota Y, et al. FDG-PET in T-cell and NK-cell neoplasms. Anal Oncol 2007;18:1685-1690.

\title{
Management of Brain Abscesses in Children Treated for Acute Lymphoblastic Leukemia
}

\author{
Herwig Lackner, MD, ${ }^{1 *}$ Petra Sovinz, MD, ${ }^{1}$ Martin Benesch, MD, ${ }^{1}$ Freyja Smolle-Jüttner, $\mathrm{MD}^{2}{ }^{2}$ Michael Mokry, MD, ${ }^{3}$ \\ Wolfgang Schwinger, $M D^{1}{ }^{1}$ Andrea Moser, $\mathrm{MD}^{1}{ }^{1}$ and Christian Urban, $\mathrm{MD}^{1}$
}

Brain abscesses in children with leukemia or other malignancies are rare and potentially fatal. We report on four children who developed brain abscesses during treatment for acute lymphoblastic leukemia $(\mathrm{ALL})$. All patients received multimodal broad-spectrum antibiotic therapy and liposomal amphotericin-B in combination with hyperbaric oxygen. First-line antimicrobial treatment was modified when a causative organism was isolated. All four patients survived, with two patients showing complete resolution of neurological and MRI abnormalities and with two patients still having residual lesions. To date, all patients are in remission with three patients still receiving antileukemic therapy. Brain abscesses can be successfully managed by a multimodality approach even in severely immunocompromised cancer patients. Pediatr Blood Cancer 2009;52:408-411. (c) 2008 Wiley-Liss, Inc.

Key words: acute lymphoblastic leukemia; brain abscesses; children; management

\section{INTRODUCTION}

Brain abscesses are rare in immunocompromised patients, and mortality rates exceeding $90 \%$ have been reported [1-8]. Only few cases of brain abscesses in children treated for acute lymphoblastic leukemia (ALL) have been reported [9-14]. Since prognosis was very dismal and little information about the appropriate management of this complication was given, we are reporting on the successful management of four children with ALL, who developed brain abscesses during antileukemic therapy.

\section{CASE REPORTS}

From January 2001 to February 2008, 68 patients were diagnosed with ALL at our institution. These children were treated according to the international treatment protocols ALL BFM 2000 or INTERFANT 06. A written informed consent was obtained from parents or their legal guardians. Supportive care included insertion of a central venous line, infection prophylaxis with oral amphotericin B, trimethoprim/sulphametoxazole $(4 / 20 \mathrm{mg} / \mathrm{kg}$, 4 times weekly), and broad spectrum antibiotics or blood products if necessary. Four children (three males and one female) developed brain abscesses during antileukemic therapy. Their ages at diagnosis of ALL were 15, 2, 4, and 0.08 years, respectively. Routine cultures of the cerebrospinal fluid (CSF) were negative in all cases. Brain abscesses occurred during induction therapy in three patients and during reinduction therapy in one patient. At onset of neurologic symptoms, lumbar puncture and magnetic resonance imaging (MRI) were performed [15]. When diagnosis of brain abscesses was confirmed, primary treatment included empirical broad spectrum antibiotics and liposomal amphotericin B (LAmB). Additionally, all children underwent treatment with hyperbaric oxygen $(\mathrm{HBO})$ as previously described $[16,17]$. Primary treatment

Additional Supporting Information may be found in the online version of this article.

${ }^{1}$ Division of Pediatric Hematology/Oncology, Department of Pediatrics and Adolescent Medicine, Medical University of Graz, Graz, Austria; ${ }^{2}$ Division of Thoracic and Hyperbaric Surgery, Medical University of Graz, Graz, Austria; ${ }^{3}$ Department of Neurosurgery, Medical University of Graz, Graz, Austria

*Correspondence to: Herwig Lackner, Division of Pediatric Hematology/Oncology, Department of Pediatrics and Adolescent Medicine, Medical University of Graz, Auenbruggerplatz 30, A-8036 Graz, Austria. E-mail: herwig.lackner@meduni-graz.at

Received 2 July 2008; Accepted 12 September 2008 